\title{
La sostenibilidad ambiental como eje transversal en la formación del diseñador gráfico
} Environmental sustainability as a transversal axis in the formation of the graphic designer

Miriam Isabel Meléndez Sandoval

Universidad de San Carlos de Guatemala https://orcid.org/0000-0003-1066-5359 Isabel.meléndez@farusac.edu.gt

\section{$\triangle$ Referencia del artículo}

Meléndez Sandoval, M. I. (2021). La sostenibilidad ambiental como eje transversal en la formación del diseñador gráfico. Revista Guatemalteca de Cultura. 1(2), 1-6.

DOI 10.46954/revistaguatecultura.v1i2.9

Recibido 15/01/2021

Aceptado 22/06/2021

\section{Resumen}

En este artículo se sintetizan los resultados de la investigación realizada en la Escuela de Diseño Gráfico de la USAC. Esta inquietud surge porque a pesar de que la Sostenibilidad Ambiental -SA- es un criterio inherente al desempeño del diseñador, es poco abordado como eje Transversal en su formación. A partir, de lo anterior, se expondrán los aspectos relevantes que sirvieron para dar respuesta al objetivo general, orientado a establecer qué lineamientos académicos son pertinentes para que en la Escuela de Diseño Gráfico, se elabore una propuesta en la que se definan las estrategias pedagógicas que permitirían que se aplique la SA de manera trasversal. 
Asimismo, se plantea que los objetivos específicos sirvieron para, indagar y documentar con docentes del curso de Visual 8 el nivel de implementación de los criterios de SA como requerimiento en los proyectos realizados y si las estrategias pedagógicas empleadas, los evidencian como eje transversal; y si las directrices teórico-prácticas serán guía, para que en la Escuela, construya una propuesta de estrategias pedagógicas que implementen los criterios de la SA como eje transversal de la formación del diseñador.

A partir de los resultados obtenidos se genera apertura por parte de la comunidad educativa proporcionando las directrices conceptuales y pragmáticas que se determinaron en el estudio, así los docentes construirán un planteamiento pedagógico que facilite que los criterios de la SA sean eje transversal en la carrera.

Palabras clave: estrategias pedagógicas, sostenibilidad, transversalidad

\section{Abstract}

This article summarizes the results of the research carried out at the USAC School of Graphic Design. This concern arises because despite the fact that Environmental Sustainability -SA- is an inherent criterion of the designer's performance, it is little approached as a transversal axis in his training. Based on the above, the relevant aspects that served to respond to the general objective will be exposed, aimed at establishing what academic guidelines are relevant so that in the School of Graphic Design, a proposal is elaborated in which the pedagogical strategies are defined that would allow the SA to be applied in a transversal manner.

Likewise, it is stated that the specific objectives were used to investigate and document with teachers of the Visual 8 course the level of implementation of the SA criteria as a requirement in the projects carried out and if the pedagogical strategies used show them as a transversal axis; and if the theoretical-practical guidelines will be a guide, so that the School builds a proposal of pedagogical strategies that implement the SA criteria as a transversal axis of the designer's training.

From the results obtained, openness is generated by the educational community by providing the conceptual and pragmatic guidelines that were determined in the study, so the teachers will build a pedagogical approach that facilitates that the SA criteria are a transversal axis in the career.

Keywords: pedagogical strategies, sustainability, transversality 


\section{Introducción}

En la enseñanza de la carrera de Diseño Gráfico el modelo utilizado es el "Modelo por Competencias" en este, los docentes contemplan lineamientos que activan el pensamiento creativo y critico del estudiante a través del desarrollo de proyectos en los que resuelve problemas de comunicación gráfica y visual, además de fortalecer elementos como trabajo en equipo, planificación del tiempo, toma de decisiones, etc.

Bajo el criterio de la enseñanza del diseño, experiencia personal y profesionalización del diseño gráfico se ve la necesidad de implementar estrategias pedagógicas dirigidas a la implementación de la sostenibilidad ambiental como un eje transversal en la Escuela de Diseño Gráfico por ser el cuidado del Medio Ambiente uno de los fundamentos filosóficos de la Universidad de San Carlos de Guatemala; con este modelo de aprendizaje los estudiantes desarrollan proyectos que los hacen más integrales en la toma de decisiones como lo es la sostenibilidad, ya que los tres ejes de estás son, el Ambiente, la Sociedad y la Economía, viendo estos parámetros percibimos que es inminente la puesta en práctica de estrategias pedagógicas que lleven a los estudiantes de la USAC a desarrollar el diseño gráfico con fines sociales, en los que contemple el beneficio de los usuarios y que además el resultado sea amigable con el ambiente, logrando de esta manera que el producto desarrollado pueda ser adquirido para el grupo de personas para quienes fue diseñado.

El problema identificado plantea el poco uso de estrategias pedagógicas que orienten los proyectos realizados a encontrar soluciones gráficas que sean sostenibles ambientalmente hablando y que además se implementen como eje transversal en la carrera de Diseño Gráfico. Según los resultados obtenidos existe un alto índice que no utiliza estrategias pedagógicas encaminadas en utilizar estos criterios de transversalidad en el tema de Sostenibilidad Ambiental.

Luego de estos hallazgos, se describirá la importancia de la Sostenibilidad Ambiental en la educación y este criterio es apoyado por el estudio y publicación Novo, (2009), Cátedra de UNESCO de Educación Ambiental y Desarrollo Sostenible, en donde se hace referencia a un cambio de mirada que contrarresta los efectos negativos de la globalización económica y el valor de fomentar ese cambio en niños, jóvenes y adultos.

Con el único fin de educar ambientalmente hablando a la población, considerando que en las cátedras universitarias se tiene una gran responsabilidad ética, moral y ambiental de la sostenibilidad y la transversalidad en el desarrollo académico del individuo frente a el lado profesional y económico, y cómo éste influye en el ámbito laboral.

Luego de identificar la problemática se realizará una descripción en la que se percibe el poco uso de las "Estrategias Pedagógicas", que orienten al estudiante en el desarrollo de la carrera universitaria, dirigiendo los proyectos de forma sostenible y transversalmente a lo largo de su aprendizaje.

Los resultados evidencian, que para el $78 \%$ de los profesores, los estudiantes demuestran un conocimiento regular o nulo del tema, esto implica que los proyectos realizados por los estudiantes no poseen en su mayoría los criterios filosóficos y ambientales que la 
Universidad requiere; Política ambiental de la Universidad de San Carlos de Guatemala, 2014, "como única universidad pública, está llamada a divulgar la cultura, ampliar los conocimientos científicos y tecnológicos, servir a la sociedad como ejemplo en el uso de los recursos naturales, así como el adecuado uso del entorno, para garantizar la sostenibilidad del desarrollo y del medio ambiente en Guatemala," por tal razón es inminente reforzar el tema en la formación del Diseñador.

\section{Materiales y métodos}

Esta investigación está orientada en un diseño descriptivo ya que se recolectaron datos por medio de encuestas en un solo momento y único tiempo, con el fin de presentar la situación encontrada con respecto al tema de interés. También tiene un enfoque de investigación aplicada, de acuerdo a lo que Vargas, (2009) afirma que este tipo de investigación es un estudio científico orientado a resolver problemas de la vida cotidiana o a controlar situaciones prácticas siendo una de sus formas "la que incluye cualquier esfuerzo sistemático y socializado por resolver problemas o intervenir en situaciones reales.

El material empleado, consiste en la elaboración de cuestionarios tipo encuestas, realizadas a docentes de la Carrera de Diseño Gráfico en relación a las Estrategias Pedagógicas en la enseñanza de la Sostenibilidad Ambiental como Eje transversal en la enseñanza del diseño, los cuales conforman una muestra total de catorce docentes quienes corresponden a los ocho talleres de Diseño Visual y el curso de Ambiente y Diseño.

\section{Resultados}

Figura 1. Representación del conocimiento que los docentes evidencian en los estudiantes sobre las SA en forma porcentual.

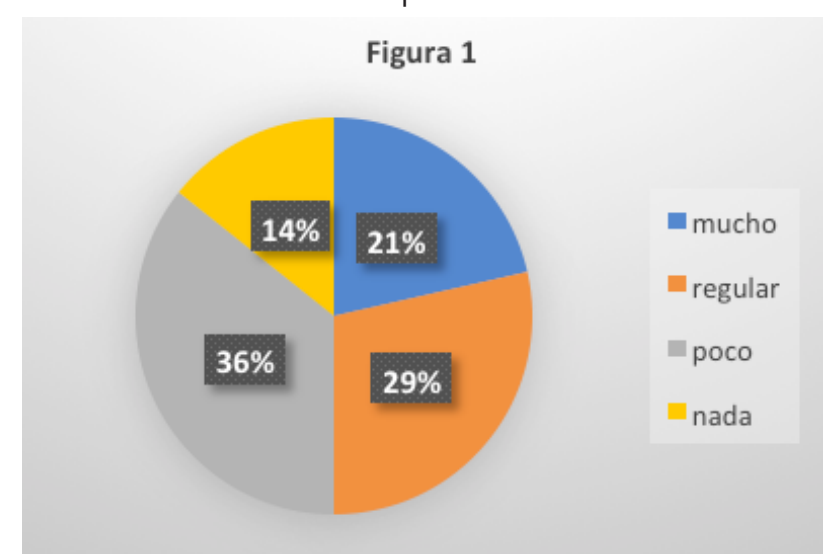

Fuente: elaboración propia mayo 2019

Para los docentes los estudiantes afirman tener conocimiento de Sostenibilidad Ambiental, en un $21 \%$ el resto de la muestra poseen poco conocimiento o nada en un $36 \%$ y $14 \%$ respectivamente.

Figura 2. Implementación por el docente de las estrategias pedagógicas en relación al tema de la SA dirigidas a los proyectos de los estudiantes, evaluadas en forma porcentual.

Figura 2

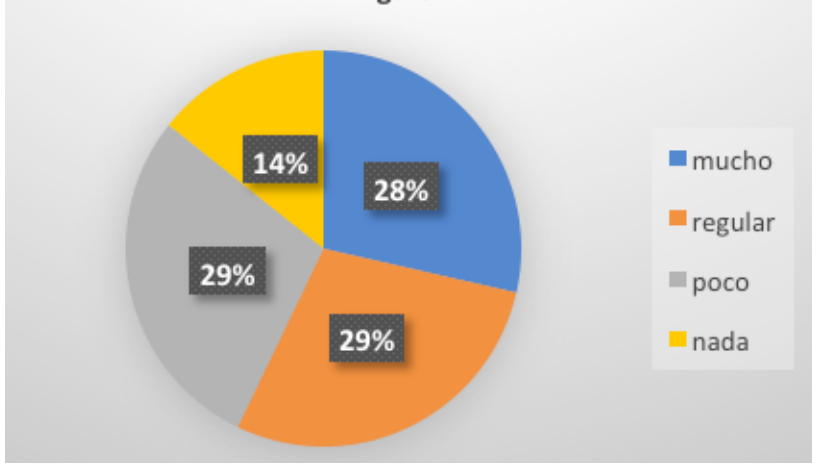

Fuente: elaboración propia mayo 2019 
El 28\% de los encuestados afirman implementar estrategias pedagógicas con el tema de la SA, mientras el $29 \%$ coinciden con aplicarlas de forma regular o poca implementación y un $14 \%$ no las implementan.

Figura 3. Representación porcentual de la aplicación de la SA como eje transversal en la carrera de Diseño Gráfico.

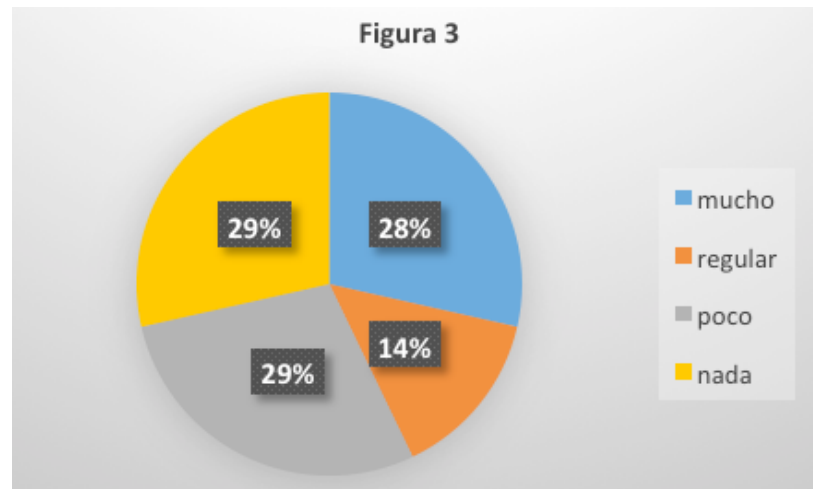

Fuente: elaboración propia mayo 2019

La SA a pesar de ser una política ambiental en la Universidad no está considerada desde una visión transversal en la malla curricular de Diseño Gráfico dato que se evidencia con un $29 \%$, poco en $29 \%$ y nada $29 \%$, se toma como una iniciativa personal más no como una estrategia pedagógica.

\section{Discusión}

A partir de la investigación se establece como lineamientos académicos para implementación de los criterios de la SA como eje transversal en la formación del diseñador gráfico de la Escuela de Diseño Gráfico, dos premisas fundamentales: primero; favorecer la realización de actividades pedagógicas que permitan la sensibilización por parte del equipo docente para que se asuma la visión ambiental propuesta por la
Universidad como criterio de formación del estudiante y segundo; desarrollar un programa de capacitación orientado a la integración progresiva de estrategias pedagógicas que respondan al nivel de evolución del estudiante a lo largo de la carrera.

Se pudo identificar que al momento de llevar a cabo la investigación, los docentes de la asignatura de Diseño Visual 8, solamente realizan actividades didácticas aisladas que no necesariamente son estrategias pedagógicas que tengan como fin el apoyo de la transversalidad de la SA en la formación del diseñador, a pesar de ello estas constituyen una oportunidad para rescatar esas iniciativas y generar una propuesta pedagógica que permita que los principios medio ambientales se apliquen en el desarrollo de proyectos de diseño de manera intencional e imperativa, respondiendo así a la responsabilidad del diseñador en el contexto contemporáneo.

Con respecto a los criterios de SA se pudo establecer dos líneas de pensamiento, una que depende del estudiante que se está formando como diseñador y otra cómo este percibe al mercado guatemalteco con respecto al tema. En el primer caso, lo que se evidencian son acciones que tienen en cuenta el cuidado del medio ambiente a nivel económico, social o ambiental de manera aislada o parcial en cuanto a los procesos de producción y reproducción de los proyectos realizados, sin embargo, no se asumen como criterios de sostenibilidad permanentes porque no se comprende su implicación al momento de aplicarlos en cada proyecto de diseño. 


\section{Referencias}

Novo, M.(2009). Revista de Educación. La educación ambiental, una genuina educación para el desarrollo sostenible 195-217

Política Ambiental de la Universidad de San Carlos de Guatemala (2014): Aprobada por el Consejo Superior Universitario en sesión celebrada el 30 de julio de 2014 Punto Sexto, Inciso 6.2 Acta 13-2014

Vargas, Z. (2009). La Investigación Aplicada: Una forma de conocer las realidades con evidencia científica. Revista Educación, 33 (1), 155165

\section{Sobre la autora}

Miriam Isabel Meléndez Sandoval, Licenciada en Diseño Gráfico, docente de la Escuela de Diseño Gráfico y estudiante de Maestría en Docencia Universitaria de la Facultad de Humanidades de la Universidad de San Carlos de Guatemala.

\section{Copyright (c) Miriam Isabel Meléndez Sandova}

Este texto está protegido por una licencia Creative Commons 4.0.

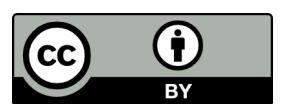

Usted es libre para compartir, copiar y redistribuir el material en cualquier medio o formato y adaptar el documento, remezclar, transformar y crear a partir del material para cualquier propósito, incluso comercialmente, siempre que cumpla la condición de atribución: usted debe reconocer el crédito de una obra de manera adecuada, proporcionar un enlace a la licencia, e indicar si se han realizado cambios. Puede hacerlo en cualquier forma razonable, pero no de forma tal que sugiera que tiene el apoyo del licenciante o lo recibe por el uso que hace. 